\title{
On the Counterpropagation of Waves
}

\author{
Roger Pizzato Nunes*10, Gunther J. L. Gerhardt ${ }^{2}$, Felipe Barbedo Rizzato ${ }^{3}$ \\ ${ }^{1}$ Universidade Federal do Rio Grande do Sul, Departamento de Engenharia Elétrica, Escola de Engenharia, RS, Brasil \\ ${ }^{2}$ Universidade de Caxias do Sul, Departamento de Física e Química, Centro de Ciências Exatas e Tecnologia, Caxias do Sul, \\ RS, Brasil \\ ${ }^{3}$ Universidade Federal do Rio Grande do Sul, Instituto de Fisica, Porto Alegre, RS, Brasil
}

Received on August 14, 2018; Revised on September 02, 2018; Accepted on September 04, 2018.

\begin{abstract}
This work analyses the counterpropagation of transversal plane waves in a linear, homogeneous, nondispersive, and isotropic medium. Although this is a traditional subject in disciplines associated with wave phenomena, a propagation analysis is not found in the literature. With this purpose, the resultant wave and consecutively its phase velocity in such system are obtained analytically. Instead of what happens with the individual waves, the analytical results demonstrate that the phase velocity of the resultant wave actually is not a constant and depends explicitly of the time or the spatial coordinates, and principally depends of the amplitudes of the individual waves. This last remarkable feature, when considering as example electromagnetic systems, can be used to adequately control and accelerate particles in a charged medium. Full agreement between the analytical and numerical results is found.
\end{abstract}

Keywords: waves, propagation, phase velocity

\section{Introduction}

The known physics can be essentially reduced and divided in two branches: one associated with particle phenomena and the other involving wave phenomena. The wave phenomena permeate many areas of physics such as mechanics, thermodynamics, electromagnetism, relativity, and quantum mechanics. One important theme associated with wave phenomena is propagation [1,2]. Essentially, wave propagation can be affected by the changing of the physical medium properties (for example, in electrodynamics the electrical permittivity $\epsilon$, the magnetic permeability $\mu$, among others), by the changing of the physical medium geometry (the changing in boundaries or the inclusion of another, as obstacles), by the existence of another waves, or by the existence of the matter. 3 Understanding how a wave propagates in a given system is of fundamental importance to understand the physics it represents.

Every propagation analysis initiates obtaining what it is called the wave vector $\boldsymbol{\beta}$ for a given angular frequency $\omega$ in a given physical system. Technically, the propagation analyses initiates with the determination of the dispersion relation $\omega(\boldsymbol{\beta})$ of the waves in the physical system. Once the dispersion relation is obtained, it becomes relevant to know how fast the wave propagates in the physical system. In other words, it is necessary to determine the velocity of the waves in the physical system.

One typical problem involving wave propagation is the case of waves propagating in opposite directions in

*Endereço de correspondência: roger.pizzato@ufrgs.br the same physical medium. Such problem is graphically shown in Figure 1 in which snapshots in times $t_{o}$ (in blue) and $t_{1}$ (in red) are taken for two arbitrary waves $\boldsymbol{\psi}_{+}$and $\boldsymbol{\psi}_{-}$. In this Figure, the wave $\boldsymbol{\psi}_{+}$propagates in the $-\mathbf{r}$ direction and the wave $\boldsymbol{\psi}_{-}$propagates in $+\mathbf{r}$ direction. One can say that wave $\boldsymbol{\psi}_{-}$counterpropagates the wave $\boldsymbol{\psi}_{+}$. The interest in this problem resides in analyzing the resultant wave $\boldsymbol{\psi}$ in the physical medium. Counterpropagating waves are present in many applied and theoretical investigations. Some applied researches involve the application of counterpropagating waves for manoeuvring, 44,5] to manipulate objects in the microscale, [6] and in fiber optics for telecommunication. [7] Theoretical researches are associated with the breaking of symmetry in nonlinear resonators, [8] wave attraction in resonant systems, 9] and its dynamics in forced systems. [10]

The problem involving counterpropagation of waves is always in focus of mechanical and electromagnetic books as a part of the propagation studies of wave phenomena. However, the approach limits itself to obtain the effective amplitude of the resultant wave as a function of characteristics of the individual waves. Reference 11 goes a little bit further and obtain the resultant wave in the medium but still just perform an analysis of the wave amplitude. Many books treat this problem still in a more restrict context, in the sense that one of the waves is a result of reflection of the other, since this configuration permeates many mechanical and electromagnetic systems, such as waves in strings of musical instruments, [12] waves in energy and communication lines, 13 to name a few examples. In this situation, dimensionless quantities like 


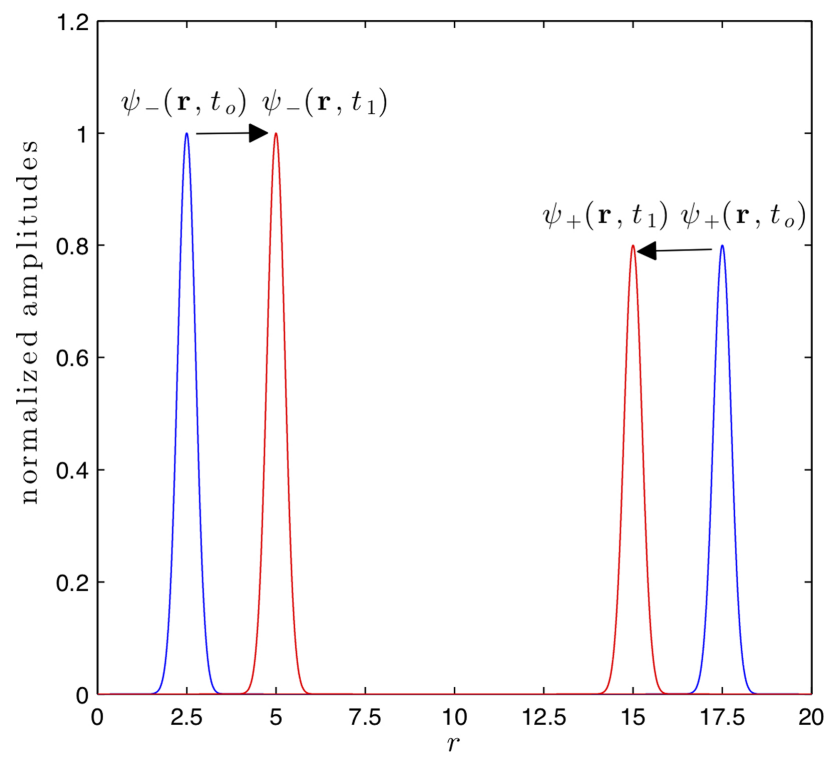

Figure 1: Snapshots in two distinct times $t_{o}$ and $t_{1}$ of two arbitrary waves $\psi_{+}$and $\psi_{-}$illustrating the contrapropagation concept. Amplitudes are normalized by the amplitude of the $\boldsymbol{\psi}_{+}$.

standing wave ratio (SWR) and the reflection coefficient acquire importance, which are associated with the amplitude of the individual waves. $14 \sqrt{16}$ However, aspects related to the propagation of the resultant wave - which are associated to the phase of the resultant wave - are not taken in to account.

Qualitatively, it is well-known that when the amplitudes of the counterpropagating waves are equal, the resultant wave presents a stationary pattern. It is merely an oscillation. It is a standing wave. But when the amplitudes of the counterpropagating waves differ, the resultant wave still presents a travelling behavior. These situations are well explored in the books in the perspective of the amplitude of the resultant wave. In the context of these systems, the SWR and the magnitude of the reflection coefficient respectively change from infinity to some finite value and from unity to some smaller value when the resultant pattern migrates from stationary to travelling. [14-16] Every literature in the field of wave phenomena stops the analysis of counterpropagating waves exactly at this point. No further discussions about the subject is presented. And from here the present work starts.

Waves are not just composed by amplitudes but also of phases. Opportunely, the existence of a phase is the unique restriction imposed by the wave equation [17] in its solution. To be a solution of the wave equation, a given arbitrary function $f$ must depend of a phase $\phi$, which relates the spatial coordinates $\mathbf{r}$ with the time $t$ in an additive way. That is, in wave phenomena, as important as to analyze the wave amplitude is to analyze the phase of the wave. The existence of a phase $\phi$ that is a combined function of the spatial coordinates $\mathbf{r}$ and the time $t$ is exactly what differs a wave from an oscillation.
In the same manner as mentioned above for the amplitude, the problem of counterpropagating waves can be explored qualitatively with a view on the phase of the resultant wave, by the means of its phase velocity. When the amplitudes of the individual waves are equal, the stationary situation, the phase velocity of the resultant wave is zero. However, when the amplitude of the individual waves differ, the phase velocity of the resultant wave is not zero. That is, the phase velocity of the resultant wave has changed between both situations, to say from zero to some finite value. Moreover, since both situations are controlled by the individual amplitudes of the counterpropagating waves, so it is the phase velocity as well. In this way, some fundamental and conceptual questions arise in this subject: what is exactly the phase velocity of the resultant wave in this system? Does the phase velocity really depend of the amplitudes of the individual waves as the simple above observation qualitatively induces? If it depends, which is the expression for the phase velocity in this system? The absence of literatures that discuss this subject and answer the questions formulated right above motivates us to develop the present work.

In this way, the purpose of the present work is to obtain the resultant wave $\boldsymbol{\psi}$ and to determine analytically its phase velocity for a system composed by two counterpropagating waves. This paper is organized as follows. In section 2 the theory associated with the phase velocity is shortly discussed. In section 3, the resultant wave in the medium is analytically determined, being its phase velocity calculated. In section 4, the analytical results are confronted with numerical simulations. In section 5 . the conclusions are presented. Finally, in section 6 , the future works, which are consequences of all shown in this paper, are presented.

\section{The theory}

Textually, the phase velocity of a given wave is defined as the velocity an observer should develop for a given reference phase of the wave seems to be static, without any relative motion between the observer and the reference point of the wave. Mathematically, defining a plane wave of amplitude $\boldsymbol{\psi}_{o}(\mathbf{r}, t)$ and phase $\phi(\mathbf{r}, t)$ as [18, 19

$$
\boldsymbol{\psi}(\mathbf{r}, t)=\boldsymbol{\psi}_{o}(\mathbf{r}, t) e^{j \phi(\mathbf{r}, t)},
$$

and supposing a reference phase $\phi_{o}=\phi\left(\mathbf{r}_{o}, t_{o}\right)$ to be followed, the phase velocity is such that in another spatial coordinate $\mathbf{r}_{1}$ in time $t_{1}$, the phase of the wave $\phi$ remais constant

$$
\phi\left(\mathbf{r}_{o}, t_{o}\right)=\phi\left(\mathbf{r}_{1}, t_{1}\right)
$$

which implies that

$$
\Delta \phi \equiv \phi\left(\mathbf{r}_{1}, t_{1}\right)-\phi\left(\mathbf{r}_{o}, t_{o}\right)=0 .
$$

The rate of equation (3) within the interval of time $\Delta t$ is

$$
\frac{\Delta \phi}{\Delta t}=0 \text {. }
$$


For equation (4) to be valid in any instant of time $t$, the following limit has to be applied

$$
\lim _{\Delta t \rightarrow 0} \frac{\Delta \phi}{\Delta t}=0,
$$

formally resulting in 18,19

$$
\frac{d}{d t} \phi(\mathbf{r}, t)=0
$$

or

$$
\frac{\partial}{\partial t} \phi(\mathbf{r}, t)+\mathbf{v}_{\mathrm{f}} \cdot \frac{\partial}{\partial \mathbf{r}} \phi(\mathbf{r}, t)=0
$$

in which $\frac{\partial}{\partial \mathbf{r}} \phi(\mathbf{r}, t)$ is the gradient of $\phi(\mathbf{r}, t)$ and $\mathbf{v}_{\mathbf{f}}$ is the velocity the observer must have to follow the reference phase of the wave, the phase velocity.

The right above equations (6) and (7) mathematically define which must be the phase velocity $\mathbf{v}_{\mathrm{f}}$ of the wave $\boldsymbol{\psi}$ specified in equation (1). For evaluating the phase velocity $\mathbf{v}_{\mathrm{f}}$, it just must be knew the phase $\phi$ of the wave $\boldsymbol{\psi}$ of equation (1). This is what will be done in the next section, in which the system of interest here, which is composed by two counterpropagating waves, will be specified and the resultant wave determined. With an analytical expression for the resultant wave $\boldsymbol{\psi}$, and consequently for its phase $\boldsymbol{\psi}$, it is possible then to determine its phase velocity.

\section{Analytical results}

The present problem consists of two counterpropagating plane waves. The plane waves considered are also uniform, which imply that its amplitudes, although might be different, are constants. One of the waves is represented by $\boldsymbol{\psi}_{+}$and the other by $\boldsymbol{\psi}_{-}$. Considering that both waves propagate in the same linear, homogeneous, and isotropic physical medium, so the resultant wave $\boldsymbol{\psi}$ in the medium is

$$
\boldsymbol{\psi}(\mathbf{r}, t)=\boldsymbol{\psi}_{+}(\mathbf{r}, t)+\boldsymbol{\psi}_{-}(\mathbf{r}, t)
$$

Assuming parallel polarization between waves, $\boldsymbol{\psi}_{+}=$ $\psi_{+} \mathbf{e}_{\psi}$ and $\boldsymbol{\psi}_{-}=\psi_{-} \mathbf{e}_{\psi}$, the equation (8) becomes scalar in the form

$$
\psi(\mathbf{r}, t)=\psi_{+}(\mathbf{r}, t)+\psi_{-}(\mathbf{r}, t)
$$

It is time to represent mathematically the individual waves $\psi_{+}$and $\psi_{-}$. Consider that the wave $\psi_{+}$is of the form

$$
\psi_{+}(\mathbf{r}, t)=\psi_{o+} e^{j(\boldsymbol{\beta} \cdot \mathbf{r}+\omega t)}
$$

in which $\psi_{o+}$ is its constant plane wave amplitude, $\boldsymbol{\beta}$ is its wave vector, and $\omega$ is its angular frequency. The counterpropagating problem is closed proposing another wave, to say $\psi_{-}$, which propagates in opposite direction of the wave $\psi_{+}$of equation 10 . This wave $\psi_{-}$can be mathematically represented in two distinct ways. One consists of flipping the signal of the spatial term of the phase $\phi$ associated with the wave $\psi_{+}$of equation 10

$$
\psi_{-}(\mathbf{r}, t)=\psi_{o_{-}} e^{j(-\boldsymbol{\beta} \cdot \mathbf{r}+\omega t)},
$$

and the other consists of flipping the signal of the temporal term of the phase $\phi$ associated with the wave $\psi_{+}$ of equation 10 )

$$
\widetilde{\psi}_{-}(\mathbf{r}, t)=\psi_{o-} e^{j(\boldsymbol{\beta} \cdot \mathbf{r}-\omega t)} .
$$

In equations 11 and 12 , $\psi_{o-}$ is the constant amplitude of the plane wave $\psi_{-}, \boldsymbol{\beta}$ is its wave vector, and $\omega$ is its angular frequency. As can be seen, the only difference that exists between the wave $\psi_{+}$and both mathematical representations of the wave $\psi_{-}$and $\widetilde{\psi}_{-}$, besides the directions of propagation, resides on its constant amplitudes.

In this way, the resultant wave $\psi$ can be represented as

$$
\psi=\psi_{o+} e^{j(\boldsymbol{\beta} \cdot \mathbf{r}+\omega t)}+\psi_{o-} e^{j(-\boldsymbol{\beta} \cdot \mathbf{r}+\omega t)},
$$

if the wave $\psi_{+}$of equation 10 and the wave $\psi_{-}$of equation (11) are inserted in equation (9), or the resultant wave $\psi$ can be represented as

$$
\widetilde{\psi}=\psi_{o+} e^{j(\boldsymbol{\beta} \cdot \mathbf{r}+\omega t)}+\psi_{o-} e^{j(\boldsymbol{\beta} \cdot \mathbf{r}-\omega t)},
$$

if the wave $\psi_{+}$of equation 10 and the wave $\psi_{-}$of equation (12) are inserted in equation (9). Although physically equivalent, both $\psi$ and $\widetilde{\psi}$ representations of the resultant wave are mathematically distinct. In this way, it can be also expected that the amplitude $\psi_{o}$ and the phase $\phi$ are also different in both representations $\psi$ and $\widetilde{\psi}$ of the resultant wave. And, as a consequence, distinct phase velocities $\mathbf{v}_{\mathrm{f}}$ will be potentially obtained in both representations.

In order to determine the phase velocity $\mathbf{v}_{\mathrm{f}}$ of the resultant wave, it is necessary to express the resultant wave, in both representations $\psi$ and $\tilde{\psi}$ right above, in the format of equation (1). When the representation $\psi$ of the resultant wave of equation 13 is chosen, one finds that its phase velocity $\mathbf{v}_{\mathrm{f}}$ is described as a function of just the spatial coordinates $\mathbf{r}$. This approach is detailed in the next subsection 3.1 and called spatial approach, due to the functional dependence of the phase velocity of the resultant wave. However, when the representation $\widetilde{\psi}$ of the resultant wave of equation (14) is chosen, one finds that its phase velocity $\widetilde{\mathbf{v}}_{\mathrm{f}}$ now can be described as a function of just the temporal coordinate $t$. This approach is treated in the next subsection 3.2 and called temporal approach, due to now the functional dependence of the phase velocity of the resultant wave to be of the time $t$. Both approaches, although mathematically distinct, are physically equivalent and can be employed to understand the propagation of the resultant wave with a spatial or temporal view of its phase velocity. This physical equivalence between both approaches is discussed in detail in the subsection 3.3 


\subsection{Spatial approach}

For expressing the resultant wave $\psi$ in the format of equation (1), one can rearrange the equation (13) as follows

$$
\psi=e^{j \omega t} \cdot\left(\psi_{o+} e^{j \boldsymbol{\beta} \cdot \mathbf{r}}+\psi_{o-} e^{-j \boldsymbol{\beta} \cdot \mathbf{r}}\right) .
$$

By the use of Euler and De Moivre relation $e^{j \theta}=\cos \theta+$ $j \sin \theta$ on the spatial terms $e^{ \pm j \boldsymbol{\beta} \cdot \boldsymbol{r}}$ of the right above equation, it is obtained

$$
\begin{aligned}
\psi & =e^{j(\omega t)} \cdot\left[\left(\psi_{o+}+\psi_{o-}\right) \cos (\boldsymbol{\beta} \cdot \mathbf{r})\right. \\
& \left.+j\left(\psi_{o+}-\psi_{o-}\right) \sin (\boldsymbol{\beta} \cdot \mathbf{r})\right]
\end{aligned}
$$

Looking for matching the equation (1) with the expression right above of equation (16), it is obtained respectively the amplitude $\psi_{o}$

$\psi_{o}(\mathbf{r})=\sqrt{\left(\psi_{o+}+\psi_{o-}\right)^{2} \cos ^{2}(\boldsymbol{\beta} \cdot \mathbf{r})+\left(\psi_{o+}-\psi_{o-}\right)^{2} \sin ^{2}(\boldsymbol{\beta} \cdot \mathbf{r})}$

and the phase $\phi$

$$
\phi(\mathbf{r}, t)=\omega t+\arctan \left[\left(\frac{\psi_{o+}-\psi_{o-}}{\psi_{o+}+\psi_{o-}}\right) \tan (\boldsymbol{\beta} \cdot \mathbf{r})\right],
$$

being the resultant wave $\psi$ of the form

$$
\psi(\mathbf{r}, t)=\psi_{o}(\mathbf{r}) e^{j \phi(\mathbf{r}, t)} .
$$

Note that the resultant amplitude $\psi_{o}$ is a function of the spatial coordinates $\mathbf{r}$ and the resultant phase $\phi$ is also a function of the time $t$. If $\psi_{o+} \rightarrow \psi_{o-}$, then $\psi_{o}(\mathbf{r}) \rightarrow 2 \psi_{o+} \cos (\boldsymbol{\beta} \cdot \mathbf{r}), \phi(\mathbf{r}, t) \rightarrow \omega t$, and consequently the resultant wave $\psi$ is merely an oscillation, representing the well-known standing wave pattern. If $\left|\psi_{o+}\right|>>\left|\psi_{o-}\right|$, then $\psi_{o}(t) \rightarrow \psi_{o+}$, and $\phi(\mathbf{r}, t) \rightarrow \boldsymbol{\beta} \cdot \mathbf{r}+\omega t$, which is essentially the $\psi_{+}$wave, as should be predicted. Similarly, if $\left|\psi_{o-}\right|>>\left|\psi_{o+}\right|$, then $\psi_{o}(t) \rightarrow \psi_{o-}$, and $\phi(\mathbf{r}, t) \rightarrow$ $\boldsymbol{\beta} \cdot \mathbf{r}-\omega t$, which is essentially the $\psi_{-}$wave, as should be also expected.

The phase velocity $\mathbf{v}_{\mathrm{f}}$ is directly obtained inserting equation (18) in equation (7) resulting in

$$
\begin{aligned}
\mathbf{v}_{\mathrm{f}} & =-\frac{\omega}{\beta} \cdot\left[\left(\frac{\psi_{o+}+\psi_{o-}}{\psi_{o+}-\psi_{o-}}\right) \cos ^{2}(\boldsymbol{\beta} \cdot \mathbf{r})\right. \\
& \left.+\left(\frac{\psi_{o+}-\psi_{o-}}{\psi_{o+}+\psi_{o-}}\right) \sin ^{2}(\boldsymbol{\beta} \cdot \mathbf{r})\right] \mathbf{e}_{\beta},
\end{aligned}
$$

in which $\mathbf{e}_{\beta}=\boldsymbol{\beta} / \beta$. One can directly verify that the phase velocity $\mathbf{v}_{\mathrm{f}}$ is a function of the spatial coordinates $\mathbf{r}$ with mean value along one wavelength $\lambda=2 \pi / \beta$

$$
\overline{\mathbf{v}}_{\mathrm{f}}=-\frac{\omega}{\beta}\left(\frac{\psi_{o+}^{2}+\psi_{o-}^{2}}{\psi_{o+}^{2}-\psi_{o-}^{2}}\right) \mathbf{e}_{\beta} .
$$

It can be also observed that the phase velocity is indeed function of the individual wave amplitudes $\psi_{o+}$ and $\psi_{o_{-}}$, as the previous qualitative analysis in Section 1- and that was the reason of this work - has predicted. If $\psi_{o+} \rightarrow \psi_{o-}$, thus $\mathbf{v}_{\mathrm{f}} \rightarrow \infty$ (and also $\left.\overline{\mathbf{v}}_{\mathrm{f}} \rightarrow \infty\right)$, which is the phase velocity of the standing wave if one considers the phase in equation (18), which in this case $\phi(\mathbf{r}, t) \rightarrow \omega t$, being $\boldsymbol{\beta}=0$, as previously observed. If $\left|\psi_{o+}\right|>>\left|\psi_{o-}\right|$, then $\mathbf{v}_{\mathrm{f}} \rightarrow-\frac{\omega}{\beta} \mathbf{e}_{\beta}$ (and also $\overline{\mathbf{v}}_{\mathrm{f}} \rightarrow-\frac{\omega}{\beta} \mathbf{e}_{\beta}$ ), which is the phase velocity of the $\psi_{+}$wave, as expected. Finally, if $\left|\psi_{o-}\right|>>\left|\psi_{o+}\right|$, then $\mathbf{v}_{\mathrm{f}} \rightarrow \frac{\omega}{\beta} \mathbf{e}_{\beta}$ (and also $\overline{\mathbf{v}}_{\mathrm{f}} \rightarrow \frac{\omega}{\beta} \mathbf{e}_{\beta}$ ), which is the phase velocity of the $\psi_{-}$wave, as would be also expected.

The Figure 2 graphically illustrates the analytical results for the resultant wave $\psi$, the phase $\phi$, and the phase velocity $v_{\mathrm{f}}$ obtained in this section. It has been considered dimensionless amplitudes $\psi_{o+}=0.1$ and $\psi_{o-}=1$, angular wave number $\beta=1 \mathrm{rad} / \mathrm{m}$, and angular frequency $\omega=1 \mathrm{rad} / \mathrm{s}$. Panel (a) of Figure 2 illustrates the propagation of the resultant wave $\psi$ of equation 19 . In this panel, the resultant wave $\psi$ is plotted along the spatial coordinates $r$ for 3 distinct times, $t=0 \mathrm{~s}, t=0.1 \mathrm{~s}$, and $t=0.2 \mathrm{~s}$. Panel (b) of this same figure presents just the phase $\phi$ of equation (18) along the spatial coordinates for these same times. Finally, the panel (c) of this figure shows the amplitude-dependance of the phase velocity $v_{\mathrm{f}}$ of equation 20 along the spatial coordinates. In this last panel, besides the previously mentioned value of $\psi_{o+}$, it has been also considered $\psi_{o+}=0.2$, and $\psi_{o+}=0.3$. One can observe in this last panel that the phase velocity always satisfies $v_{\mathrm{f}}>0$, since for each case $\psi_{o+}<\psi_{o-}$, being the direction of propagation thus governed by the wave $\psi_{-}$.

A final analysis of the analytical results of this section must confront the behaviour of the phase velocity plotted in Figure 2(c) with the propagation of the resultant wave plotted in Figure Figure 2(a). For that, consider the spatial coordinates comprised in the interval $1.5 \lesssim r \lesssim 3$. Looking the phase velocity $v_{\mathrm{f}}$ for $\psi_{\mathrm{o}+}=0.1$, the blue

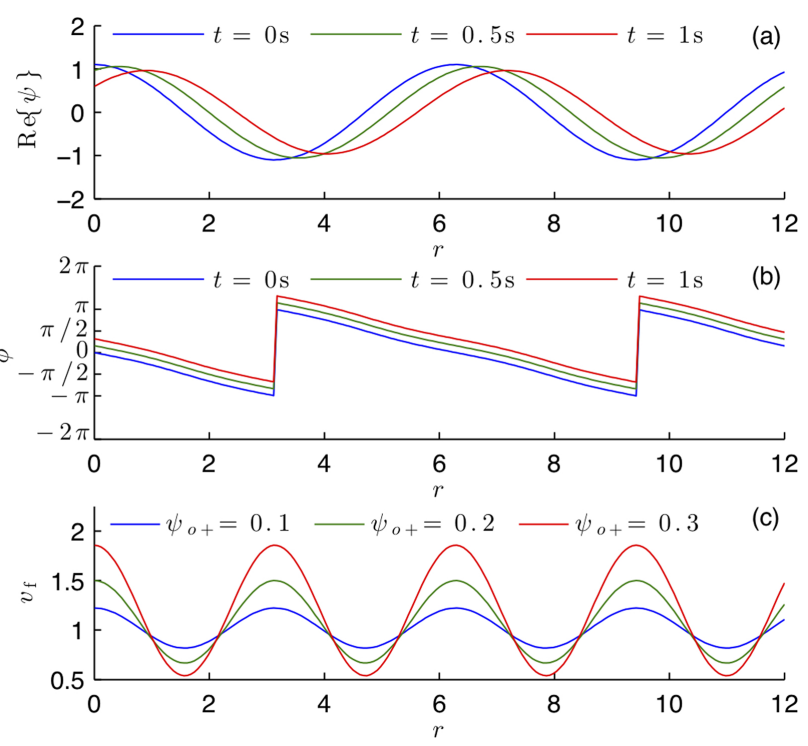

Figure 2: Graphical illustration of (a) the resultant wave $\psi$, (b) the phase $\phi$, and (c) the phase velocity $v_{\mathrm{f}}$. 
curve of Figure 2(c), one can observe that $v_{\mathrm{f}}$ increases with the increase of $r$. This behaviour of $v_{\mathrm{f}}$ is in full accordance with what is observed in Figure 2(a), in which, for a phase reference that turns $\psi=0$, it can be observed, as time evolves, that the distance between the spatial coordinates in which $\psi=0$ also increases. In this way, since the interval of time between the snapshots of the resultant wave is constant, then the phase velocity of the resultant wave are also increasing in this interval.

The next subsection will treat of the second approach, which is associated with the representation $\psi$ of the resultant wave described by equation (14).

\subsection{Temporal approach}

Instead of what has been performed in the previous section 3.1 , evidencing the term $e^{j(\boldsymbol{\beta} \cdot \mathbf{r})}$ in equation (14) it is possible to obtain

$$
\widetilde{\psi}=e^{j(\boldsymbol{\beta} \cdot \mathbf{r})} \cdot\left(\psi_{o+} e^{j \omega t}+\psi_{o-} e^{-j \omega t}\right),
$$

which becomes

$$
\begin{aligned}
\widetilde{\psi} & =e^{j(\boldsymbol{\beta} \cdot \mathbf{r})} \cdot\left[\left(\psi_{o+}+\psi_{o-}\right) \cos (\omega t)\right. \\
& \left.+j\left(\psi_{o+}-\psi_{o-}\right) \sin (\omega t)\right],
\end{aligned}
$$

when the Euler and De Moivre relation is applied to the temporal terms $e^{ \pm j \omega t}$ and some algebra is performed.

From the matching of equation (1) with the expression between the squared brackets of equation (23), it is obtained respectively the amplitude $\widetilde{\psi}_{o}$

$\widetilde{\psi}_{o}(t)=\sqrt{\left(\psi_{o+}+\psi_{o-}\right)^{2} \cos ^{2}(\omega t)+\left(\psi_{o+}-\psi_{o-}\right)^{2} \sin ^{2}(\omega t)}$

and the phase $\phi$

$$
\widetilde{\phi}(\mathbf{r}, t)=\boldsymbol{\beta} \cdot \mathbf{r}+\arctan \left[\left(\frac{\psi_{o+}-\psi_{o-}}{\psi_{o+}+\psi_{o-}}\right) \tan (\omega t)\right],
$$

being the resultant wave $\widetilde{\psi}$ of the form

$$
\widetilde{\psi}(\mathbf{r}, t)=\widetilde{\psi}_{o}(t) e^{j \widetilde{\phi}(\mathbf{r}, t)} .
$$

Note that the resultant amplitude $\widetilde{\psi}_{o}$ is a function of time $t$ and the resultant phase $\widetilde{\phi}$ is also a function of space coordinates $\mathbf{r}$. If $\psi_{o+} \rightarrow \psi_{o-}$, then $\widetilde{\psi}_{o}(t) \rightarrow 2 \psi_{o+} \cos (\omega t)$, $\widetilde{\phi}(\mathbf{r}, t) \rightarrow \boldsymbol{\beta} \cdot \mathbf{r}$, and consequently the resultant wave $\widetilde{\psi}$ is merely an oscillation, representing the well-known standing wave pattern. If $\left|\psi_{o+}\right|>>\left|\psi_{o-}\right|$, then $\widetilde{\psi}_{o}(t) \rightarrow$ $\psi_{o+}$, and $\widetilde{\phi}(\mathbf{r}, t) \rightarrow \boldsymbol{\beta} \cdot \mathbf{r}+\omega t$, which is essentially the $\psi_{+}$ wave, as should be predicted. Similarly, if $\left|\psi_{o-}\right|>>\left|\psi_{o+}\right|$, then $\widetilde{\psi}_{o}(t) \rightarrow \psi_{o-}$, and $\widetilde{\phi}(\mathbf{r}, t) \rightarrow \boldsymbol{\beta} \cdot \mathbf{r}-\omega t$, which is essentially the $\psi_{-}$wave, as should be also expected.

The phase velocity $\widetilde{\mathbf{v}}_{\mathrm{f}}$ is directly obtained inserting equation 25) in equation (7) resulting in

$$
\begin{aligned}
& \widetilde{\mathbf{v}}_{\mathrm{f}}=-\frac{\omega}{\beta} \text {. } \\
& \frac{\left(\psi_{o+}+\psi_{o-}\right)\left(\psi_{o+}-\psi_{o-}\right)}{\left(\psi_{o+}+\psi_{o-}\right)^{2} \cos ^{2}(\omega t)+\left(\psi_{o+}-\psi_{o-}\right)^{2} \sin ^{2}(\omega t)} \mathbf{e}_{\beta} .
\end{aligned}
$$

One can directly verify that the phase velocity $\widetilde{\mathbf{v}}_{\mathrm{f}}$ is a function of the time $t$ with mean value along one period $T=2 \pi / \omega$

$$
\left\langle\widetilde{\mathbf{v}}_{\mathrm{f}}\right\rangle=-\frac{\omega}{\beta} \operatorname{sign}\left(\psi_{o+}-\psi_{o-}\right) \mathbf{e}_{\beta},
$$

in which $\operatorname{sign}(x)=1$ for $x>0, \operatorname{sign}(x)=-1$ for $x<0$, and $\operatorname{sign}(x)=0$ for $x=0$.

In the same manner as performed in the previous subsection, it can be also observed that the phase velocity is indeed function of the individual wave amplitudes $\psi_{o+}$ and $\psi_{o-}$, as the previous simple qualitative analysis in Section 1 - and that was the reason of this work has predicted. If $\psi_{o+} \rightarrow \psi_{o-}$, thus $\widetilde{\mathbf{v}}_{\mathrm{f}} \rightarrow \mathbf{0}$ (and also $\left\langle\widetilde{\mathbf{v}}_{\mathrm{f}}\right\rangle \rightarrow \mathbf{0}$ ), which is the phase velocity of the standing wave. If $\left|\psi_{o+}\right|>>\left|\psi_{o-}\right|$, then $\widetilde{\mathbf{v}}_{\mathrm{f}} \rightarrow-\frac{\omega}{\beta} \mathbf{e}_{\beta}$ (and also $\left\langle\widetilde{\mathbf{v}}_{\mathrm{f}}\right\rangle=-\frac{\omega}{\beta} \mathbf{e}_{\beta}$ ), which is the phase velocity of the $\psi_{+}$wave, as expected. Finally, if $\left|\psi_{o-}\right|>>\left|\psi_{o+}\right|$, then $\widetilde{\mathbf{v}}_{\mathrm{f}} \rightarrow \frac{\omega}{\beta} \mathbf{e}_{\beta}$ (and also $\left\langle\widetilde{\mathbf{v}}_{\mathrm{f}}\right\rangle=\frac{\omega}{\beta} \mathbf{e}_{\beta}$ ), which is the phase velocity of the $\psi_{-}$wave, as would be also expected. In this way, the phase velocity $\widetilde{\mathbf{v}}_{\mathrm{f}}$ of the resultant wave can be adequately adjusted to absolute values smaller than the phase velocity $\omega / \beta$ of the individual plane waves.

As performed in the previous section, the Figure 3 graphically illustrates the analytical results for the resultant wave $\widetilde{\psi}$, the phase $\widetilde{\phi}$, and the phase velocity $\widetilde{v}_{\mathrm{f}}$ obtained in this section. It has been also considered dimensionless amplitudes $\psi_{o+}=0.1$ and $\psi_{o-}=1$, angular wave number $\beta=1 \mathrm{rad} / \mathrm{m}$, and angular frequency $\omega=1 \mathrm{rad} / \mathrm{s}$. Panel (a) of Figure 3 illustrates the propagation of the resultant wave $\widetilde{\psi}$ of equation 26 . In this panel, the resultant wave $\widetilde{\psi}$ is plotted along the time $t$ for 3 distinct spatial coordinates, $r=0 \mathrm{~m}, r=0.1 \mathrm{~m}$, and $r=0.2 \mathrm{~m}$. Panel (b) of this same figure presents just the phase $\widetilde{\phi}$ of equation 25 along the time for these same
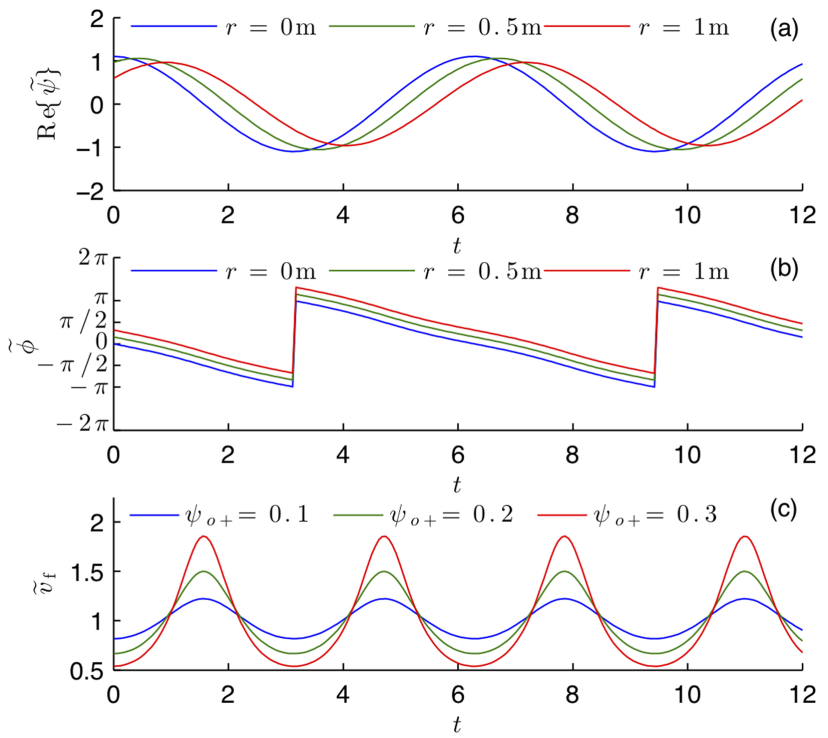

Figure 3: Graphical illustration of (a) the resultant wave $\widetilde{\psi}$, (b) the phase $\widetilde{\phi}$, and (c) the phase velocity $\widetilde{v}_{\mathrm{f}}$. 
spatial coordinates. Finally, the panel (c) of this figure shows the amplitude-dependance of the phase velocity $\widetilde{v}_{\mathrm{f}}$ of equation (27) along the time. In this last panel, besides the previously mentioned value of $\psi_{o+}$, it has been also considered $\psi_{o+}=0.2$, and $\psi_{o+}=0.3$. One can also observe in this last panel that the phase velocity always satisfies $\widetilde{v}_{\mathrm{f}}>0$, since for each of these cases $\psi_{o+}<\psi_{o_{-}}$, being the direction of propagation thus governed by the wave $\psi_{-}$.

Similarly with what has been done in the previous section, to conclude the analysis of the analytical results of this section, one must confront the behaviour of the phase velocity plotted in Figure 3(c) with the propagation of the resultant wave plotted in Figure 3(a). For that, consider the interval of time $1.5 \lesssim t \lesssim 3$. Looking the phase velocity $\widetilde{v}_{\mathrm{f}}$ for $\psi_{o+}=0.1$, the blue curve of Figure 3(c), one can observe that $\widetilde{v}_{\mathrm{f}}$ decreases with the increase of $t$. Again, this behaviour of $\widetilde{v}_{\mathrm{f}}$ in this interval of time is in full accordance with what can be seen in Figure 3(a), since the time elapsed between each crossing of $\psi$ by 0 increases. In this way, since the distance between the spatial coordinates in which the resultant wave is analysed is constant, then the phase velocity of the resultant wave are also decreasing in this interval.

\subsection{The equivalence between both approaches}

The spatial and temporal approaches presented before produced different expressions for the phase and amplitude of the resultant wave. However, it is expected that the analytical results for the wave $\psi$ of equation (19) and $\widetilde{\psi}$ of equation $(26)$, which involves the product of the resultant wave amplitudes and phases in both representations, describes absolutely the same wave, although they have different mathematical representations. Rigorously, the numerical results shown in the next section 4 comprove that the analytical equations 19 and 26 for the resultant wave in both representations are physically equivalent and are absolutely correct. However, quantities derived of just the amplitude or of just the phase (not of the product of both) of the resultant wave do not necessary match for every spatial coordinate $\mathbf{r}$ or the time $t$. This is the case of the phase velocity of the resultant wave, which is an expression derived from just the phase of the resultant wave. It is then necessary a detailed inspection of these expressions to connect the results produced by both approaches.

For evaluating compatibility between the phase velocities $\mathbf{v}_{\mathrm{f}}$ and $\widetilde{\mathbf{v}}_{\mathrm{f}}$ it is necessary that the reference in the waves $\psi$ and $\widetilde{\psi}$ adopted is the same. If the reference adopted is exactly the same in both representations, it is expected the expressions of the phase velocity in both approaches produce the same result. An adequate phase reference to choose is

$$
\phi(\mathbf{r}, t)=\widetilde{\phi}(\mathbf{r}, t)=m \pi / 2
$$

since $\operatorname{Re}\{\psi\}=\operatorname{Re}\{\widetilde{\psi}\}=0$, eliminating the influences of the different aspects of the resultant wave amplitude in both representations. $m=2 n+1$, and $n$ is an integer number. Inserting this reference phase in equation 18 results in

$$
\tan (m \pi / 2-\omega t)=\left(\frac{\psi_{o+}-\psi_{o-}}{\psi_{o+}+\psi_{o-}}\right) \tan (\boldsymbol{\beta} \cdot \mathbf{r}),
$$

while inserting in equation 25 results in

$$
\tan (m \pi / 2-\boldsymbol{\beta} \cdot \mathbf{r})=\left(\frac{\psi_{o+}-\psi_{o-}}{\psi_{o+}+\psi_{o-}}\right) \tan (\omega t) .
$$

Considering that $\tan (m \pi / 2-x)=1 / \tan (x)$, then both equations (30) and (31) produces exactly the same expression

$$
\left(\frac{\psi_{o+}-\psi_{o-}}{\psi_{o+}+\psi_{o-}}\right) \tan (\omega t) \tan (\boldsymbol{\beta} \cdot \mathbf{r})=1 .
$$

Essentially, the equation (32) establishes, for any given time $t$ in which $\phi=m \pi / 2$, which is the spatial coordinate $\mathbf{r}$ in which the $\widetilde{\phi}$ is also $m \pi / 2$. That is, this is the relation between the spatial coordinates $\mathbf{r}$ and the time $t$ to follow the same reference phase in both spatial and temporal approaches employed to represent the resultant wave. In this situation, since the reference phase adopted is the same in the approaches, it is expected that the phase velocities $\mathbf{v}_{\mathrm{f}}$ and $\widetilde{\mathbf{v}}_{\mathrm{f}}$ match its results.

The figure 4 shows the absolute value of the phase velocity $v_{\mathrm{f}}$ along the spatial coordinates $\mathbf{r}=r \mathbf{e}_{r}$. Also, in this figure, the phase velocity $\widetilde{v}_{\mathrm{f}}$ is presented. The phase velocity $\widetilde{v}_{\mathrm{f}}$ is outlined along the spatial coordinates $r$ by the means of the equation (32). For each spatial coordinate $v_{\mathrm{f}}$ is plotted, the corresponding value of the time $t$ is obtained through the equation (32) and then $\widetilde{v}_{\mathrm{f}}$ is plotted. This procedure assure that any value of $v_{\mathrm{f}}$ is compared with $\widetilde{v}_{\mathrm{f}}$ for exactly the same reference

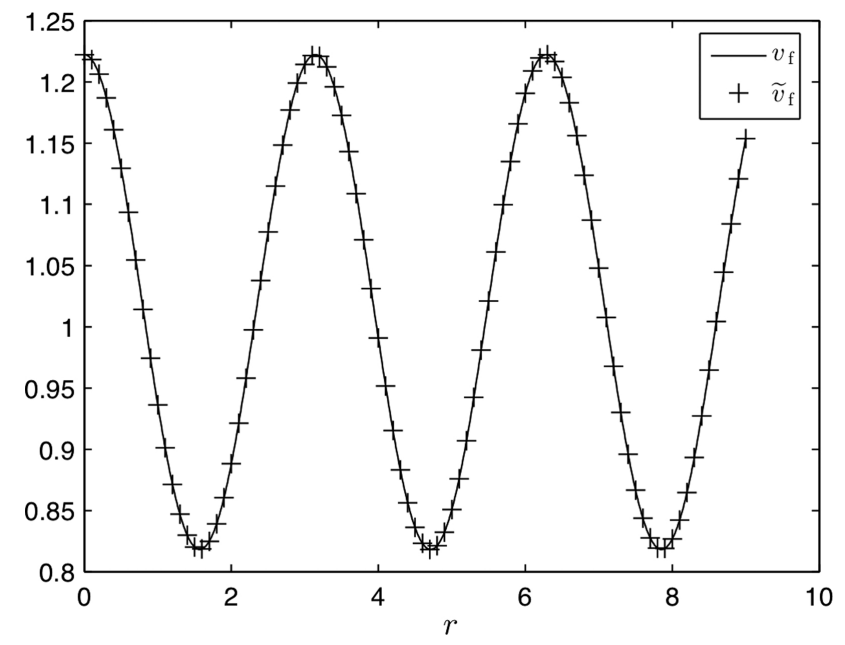

Figure 4: Comparison between the phase velocities $v_{\mathrm{f}}$, plotted as a continuous line, and $\widetilde{v}_{\mathrm{f}}$, plotted with crosses, for the same reference phase along the spatial coordinates $r$. 
phase. It can be observed a perfect agreement between both expressions of the phase velocity. For completeness, the figure 5 presents the results for the phase velocity $\widetilde{v}_{\mathrm{f}}$ along the time $t$ together with the phase velocity $v_{\mathrm{f}}$. The phase velocity $v_{\mathrm{f}}$ is determined calculating, for each time $t$, which is the corresponding spatial position $r$ through the equation (32). It can be observed again a nice agreement. In figure 4 and 5 it is adopted $\psi_{o+}=0.1$ and $\psi_{o_{-}}=1$, which are dimensionless, with $\omega=1 \mathrm{rad} / \mathrm{s}$ and $\beta=1 \mathrm{rad} / \mathrm{m}$.

The analytical expressions of both approaches satisfied the expected results in the limit situations explored above in each subsection. Also, it has been observed that both approaches are equivalent when the same reference phase of the resultant wave is considered. However, for full validation, the analytical expressions obtained for the phase velocity through both approaches must be confronted with the results obtained from the direct numerical simulation of equation (9) with equation (10) and equation (11) or equation 12). In this way, in the next section, a direct comparison between the analytical and numerical results for the resultant wave and its phase velocity will occur.

\section{Comparison with numerical simulations}

The analytical results presented in the previous section 3 are confronted here with numerical simulations of the counterpropagating waves. In Figure 6 and Figure 7, as the first comparison, it is shown the analytical and numerical results for the resultant wave $\psi$ respectively as a function of time $t$ and of spatial coordinates $\mathbf{r}=r \mathbf{e}_{r}$. The numerical results consist of the direct simulation of the real part of the resultant wave $\boldsymbol{\psi}$ described in equation (9) with the individual waves of equation $(10)$ and equations

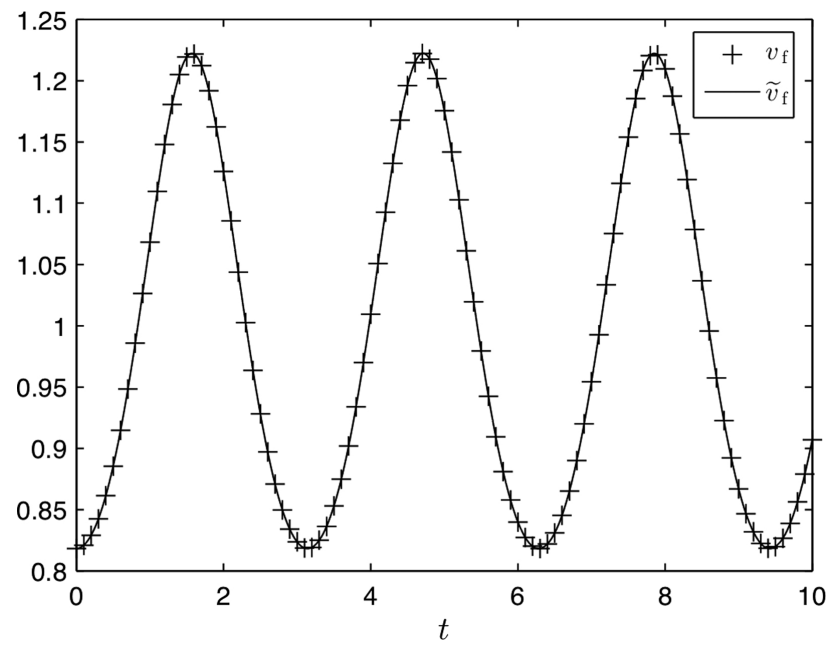

Figure 5: Comparison between the phase velocities $v_{\mathrm{f}}$, plotted with crosses, and $\widetilde{v}_{\mathrm{f}}$, plotted as a continuous line, for the same reference phase along the time $t$.

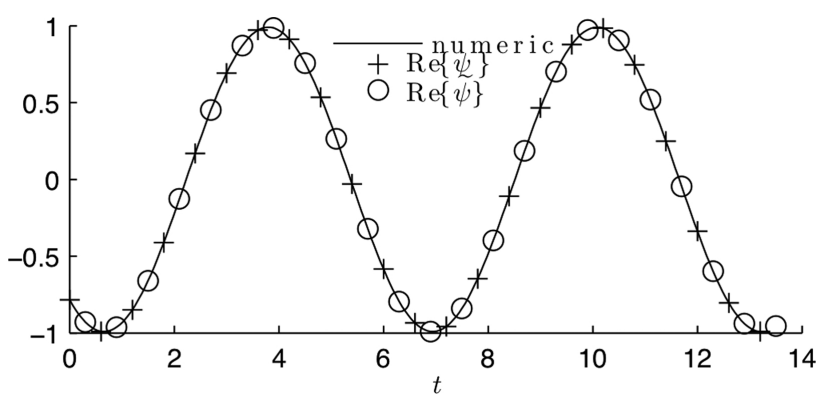

Figure 6: Comparison between the numerical and analytical results for the resultant wave $\psi$ in the medium as a function of time $t$ for $r=4 \mathrm{~m}$. The results of the spatial and temporal approaches are respectively represented with crosses and circles.

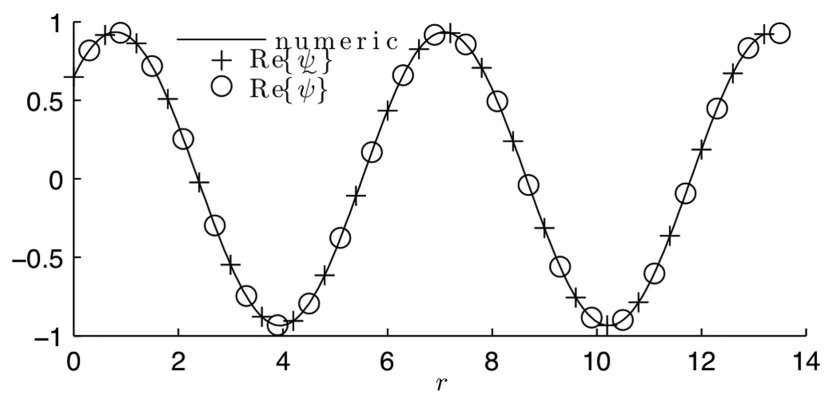

Figure 7: Comparison between the numerical and analytical results for the resultant wave in the medium as a function of spatial coordinate $r$ for $t=1 \mathrm{~s}$. The results of the spatial and temporal approaches are respectively represented with crosses and circles.

(11) or 12, depending of the representation adopted. The analytical results consist in evaluating the real part of the resultant wave of equation (19) with the resultant amplitude of equation (17) and the resultant phase of equation (18), for the spatial approach (represented with crosses), and in to evaluate the real part of the resultant wave of equation 26 with the resultant amplitude of equation (24) and the resultant phase of equation (25), in the case of the temporal approach (represented with circles). A perfect agreement with numerical simulations is achieved in both analytical approaches as expected. The dimensionless waves amplitudes are $\psi_{o+}=0.2$ and $\psi_{o_{-}}=1$, the angular wave number is $\beta=1 \mathrm{rad} / \mathrm{m}$, and the angular frequency is $\omega=1 \mathrm{rad} / \mathrm{s}$. Perfect agreement is also found for other values of waves amplitudes, wave number, and angular frequency, being satisfactory to comprove that the analytical expressions for the resultant wave $\psi$ in both approaches are correct.

The phase velocity $\mathbf{v}_{f}$ can be computed numerically choosing a specific phase $\phi_{n}(\mathbf{r}, t)$ and following it along the spatial coordinates $r$ or along the time $t$. Since wave propagates, the reference phase $\phi_{n}(\mathbf{r}, t)$ will be found in distinct and successive times $t$ as the spatial coordinates are followed. For each interval of time $\Delta t$, it is possible to calculate the displacement $\Delta r$. In other way, the reference phase $\phi_{n}(\mathbf{r}, t)$ can also be found in distinct and 
successive spatial coordinates $r$ as time $t$ evolves. For each displacement $\Delta r$, it is possible then to calculate the necessary time interval $\Delta t$. Independently of following the spatial or temporal coordinates, the absolute value of the phase velocity estimated numerically is

$$
v_{\mathrm{fn}} \approx \Delta r / \Delta t
$$

in which the subscript $n$ stands for numeric. To follow spatial or temporal coordinates will be suitable when the comparison of the numerical results for the phase velocity occurs respectively with the analytical results provided by the spatial and temporal approaches. Since the resultant wave $\psi$ is periodic, it only must be assured that $\Delta r<<2 \pi / \beta$ and $\Delta t<<2 \pi / \omega$, avoiding ambiguity problems when tracking the reference phase $\phi_{n}$ of the wave respectively along the spatial coordinates $r$ or the time $t$. This numeric procedure will be applied right below when the analytical results from both approaches will be confronted with the numerical results for the phase velocity.

Figure 8 and Figure 9 presents the results along the spatial coordinates $r$ for respectively $\psi_{o+}=0.1$ and $\psi_{o+}=0.2$ in distinct times. This is the only difference between both Figures. For both, $\psi_{o_{-}}=1, \beta=1 \mathrm{rad} / \mathrm{m}$, and $\omega=1 \mathrm{rad} / \mathrm{s}$. For simplicity, the reference phase $\phi_{n}$ chosen is such that turns the resultant wave $\psi=0$. This assumption substantially simplifies the tracking of the reference phase $\phi_{n}$ along the spatial coordinates $r$, once the amplitude of the resultant wave also depends of the spatial coordinates $r$, and it can induces to errors in following the reference phase point $\phi_{n}$. The $\psi=0$ curve is plotted together in the panels (a) of Figures 8 and 9
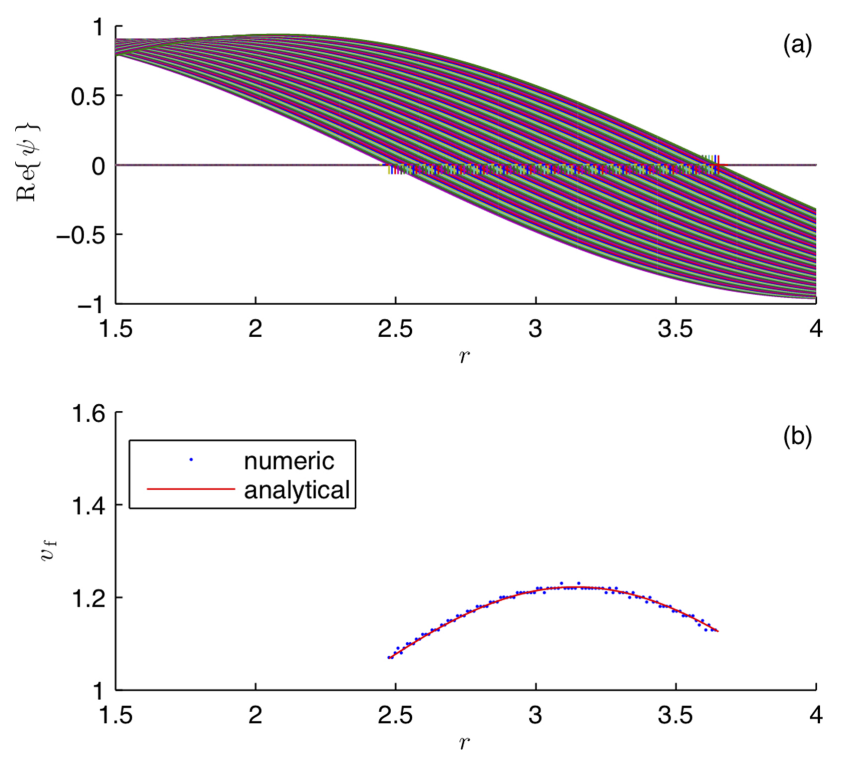

Figure 8: Cumulative plots of the resultant wave $\psi$ along the spatial coordinate $r$ for 100 successive times $t_{n}$ with $\Delta t_{n}=0.01 \mathrm{~s}$ are shown in panel (a). A comparison between the numerical and analytical results for the absolute value of the phase velocity $v_{f}$ is shown in panel (b). In both panels, $\psi_{o+}=0.1$.
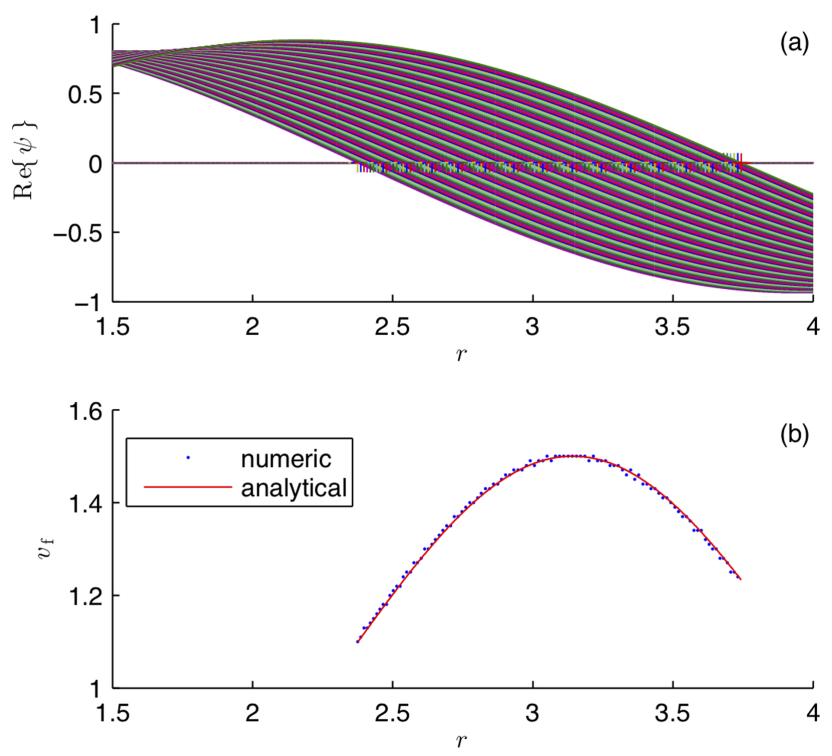

Figure 9: Cumulative plots of the resultant wave $\psi$ along the spatial coordinate $r$ for 100 successive times $t_{n}$ with $\Delta t_{n}=0.01 \mathrm{~s}$ are shown in panel (a). A comparison between the numerical and analytical results for the absolute value of the phase velocity $v_{f}$ is shown in panel (b). In both panels, $\psi_{o+}=0.2$.

for analysis purposes. The reference phase $\phi_{n}$ is tracked along $1 \mathrm{~s} \leq t_{n} \leq 2 \mathrm{~s}$, in steps of $\Delta t_{n}=0.01 \mathrm{~s}$. The resultant wave $\psi$ along the spatial coordinates $r$ for each one of these times $t_{n}$ is cumulatively plotted in the panels (a) of Figures 8 and 9 In these Figures, each color represents the resultant wave picture along the spatial coordinates $r$ in each one of the times $t_{n}$. The total number of curves plotted in the panels (a) of Figures 8 and 9 are 100, which shows to be adequate to describe the phase velocity in the interval of spatial coordinates $1.5 \mathrm{~m} \leq r \leq 4 \mathrm{~m}$ with a reasonable accuracy. The crossing by $\psi=0$ of the $\psi\left(r, t_{n}\right)$ for each time $t_{n}$ occurs in a spatial coordinate $r_{n}$ and is detached by a '+' sign. The spatial displacement $\Delta r$ of equation (33) computed numerically is exactly the spatial displacement $\Delta r_{n}$ between each consecutive '+' sign. Figure 10 details and illustrates how $\Delta r$ is evaluated numerically for the first two waves plotted in Figure 8 Figure 10 represents a zoom of Figure 8 for $-0.01 \leq$ $\psi \leq 0.05$ and $1.5 \mathrm{~m} \leq r \leq 4 \mathrm{~m}$. The interval of time $\Delta t$ of equation 33 is exactly the previously specified $\Delta t_{n}=0.01 \mathrm{~s}$, a constant. The phase velocity is numerically calculated inserting all the spatial displacement $\Delta r_{n}$ computed with $\Delta t_{n}=0.01 \mathrm{~s}$ in equation (33). Panel (b) of Figures 8 and 9 compares the numeric and analytical results for the absolute value of the phase velocity $v_{\mathrm{f}}$ for respectively the wave amplitudes $\psi_{o+}=0.1$ and $\psi_{o+}=0.2$ described above. The analytical result for the phase velocity $v_{\mathrm{f}}$ uses the expression described in equation (20). Nice agreement can be observed.

Similarly, the Figure 11 and Figure 12 presents the results for respectively $\psi_{o+}=0.1$ and $\psi_{o+}=0.2$ along the time $t$ in distinct spatial positions. This is the only 


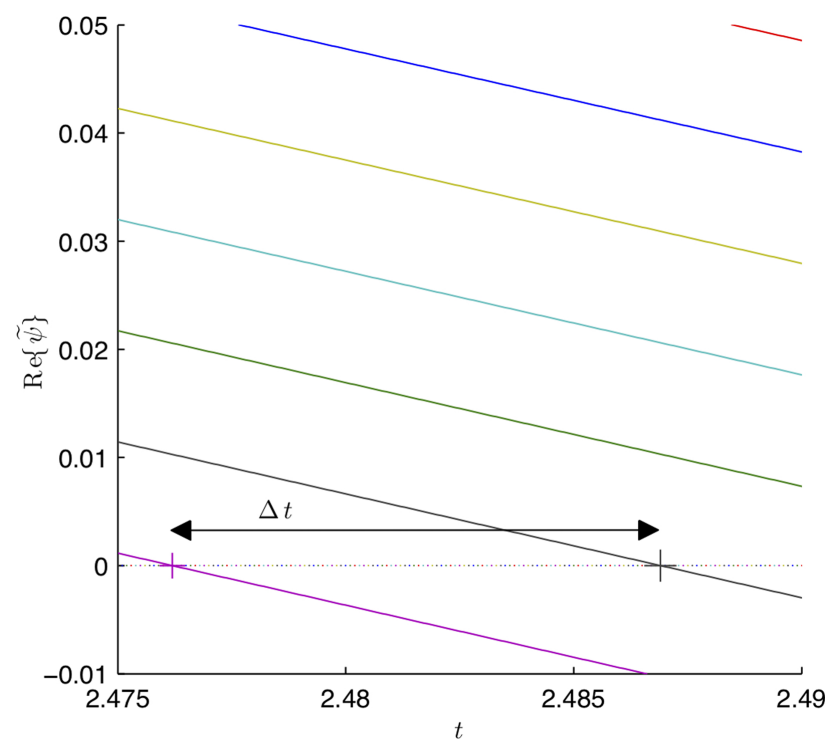

Figure 10: A detailed description of the crossing spatial coordinates by $\psi=0$ of the first two waves plotted in Figure 8 , The difference between this consecutive crossing spatial coordinates is exactly the displacement interval $\Delta r$ used to evaluate the phase velocity in this specific interval.
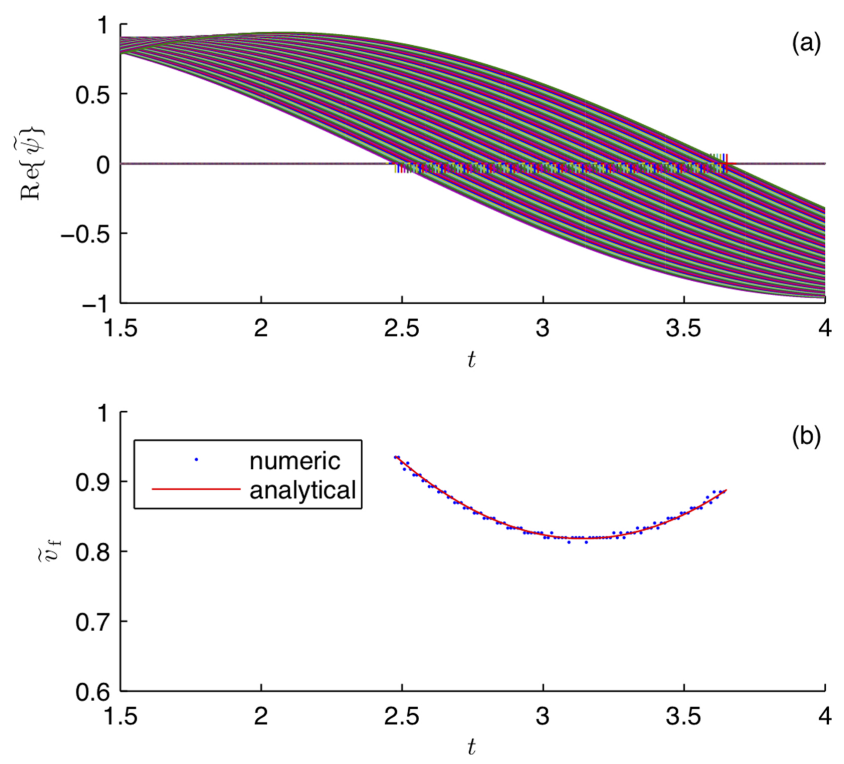

Figure 11: Cumulative plots of the resultant wave $\widetilde{\psi}$ along the time $t$ for 100 successive spatial positions $r_{n}$ with $\Delta r_{n}=0.01 \mathrm{~m}$ are shown in panel (a). A comparison between the numerical and analytical results for the absolute value of the phase velocity $\widetilde{v}_{f}$ is shown in panel (b). In both panels, $\psi_{o+}=0.1$.

difference between both Figures. For both, $\psi_{o_{-}}=1, \beta=$ $1 \mathrm{rad} / \mathrm{m}$, and $\omega=1 \mathrm{rad} / \mathrm{s}$. For simplicity, the reference phase $\phi_{n}$ chosen is such that turns the resultant wave $\psi=$ 0 . This assumption substantially simplifies the tracking of the reference phase $\phi_{n}$ along the time $t$, once the amplitude of the resultant wave also depends of the time $t$ in this approach, and it can induces to errors in following the reference phase point $\phi_{n}$. The $\psi=0$ curve
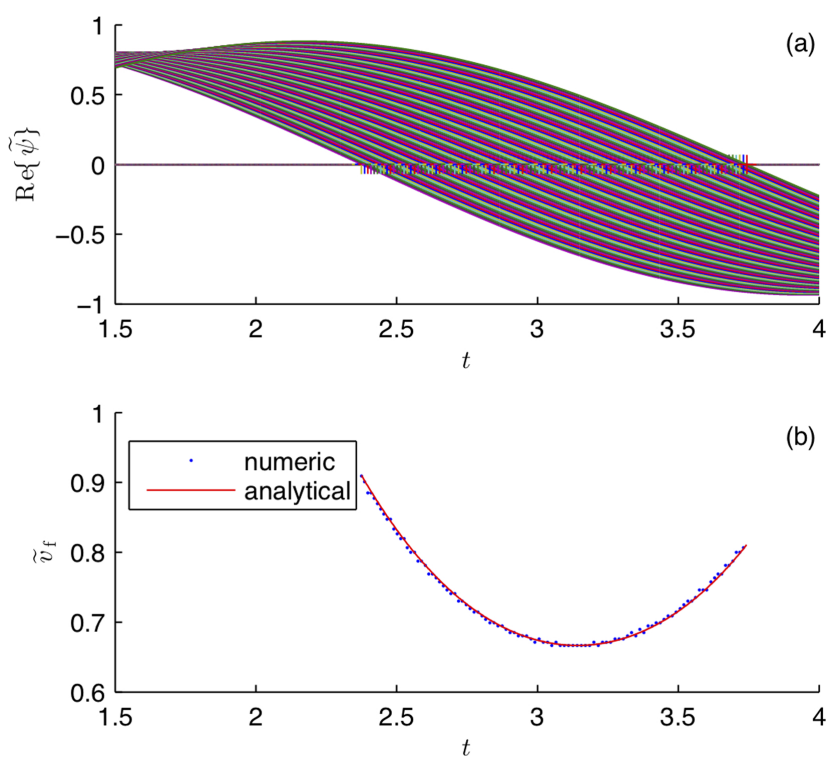

Figure 12: Cumulative plots of the resultant wave $\widetilde{\psi}$ along the time $t$ for 100 successive spatial positions $r_{n}$ with $\Delta r_{n}=0.01 \mathrm{~m}$ are shown in panel (a). A comparison between the numerical and analytical results for the absolute value of the phase velocity $\widetilde{v}_{f}$ is shown in panel (b). In both panels, $\psi_{o+}=0.2$.

is plotted together in the panels (a) of Figures 11 and 12 for analysis purposes. The reference phase $\phi_{n}$ is tracked along $1 \mathrm{~m} \leq r_{n} \leq 2 \mathrm{~m}$, in steps of $\Delta r_{n}=0.01 \mathrm{~m}$. The resultant wave $\psi$ along time $t$ for each one of these spatial coordinates $r_{n}$ is cumulatively plotted in the panels (a) of Figures 11 and 12 In these Figures, each color represents the resultant wave picture along time $t$ in each one of the spatial coordinates $r_{n}$. The total number of curves plotted in the panels (a) of Figures 11 and 12 are 100 , which shows to be adequate to describe the phase velocity in the interval of time $2.5 \mathrm{~s} \leq t \leq 3.5 \mathrm{~s}$ with a reasonable accuracy. The crossing by $\psi=0$ of the $\psi\left(r_{n}, t\right)$ for each spatial coordinate $r_{n}$ occurs in a time $t_{n}$ and is detached by a ' + ' sign. The interval of time $\Delta t$ of equation (33) computed numerically is exactly the interval of time $\Delta t_{n}$ between each consecutive '+' sign. Figure 13 details and illustrates how $\Delta t$ is evaluated numerically for the first two waves plotted in Figure 11 . Figure 13 represents a zoom of Figure 11 for $-0.01 \leq \psi \leq 0.05$ and $2.475 \mathrm{~s} \leq t \leq$ $2.49 \mathrm{~s}$. The spatial displacement $\Delta r$ of equation $(33)$ is exactly the previously specified $\Delta r_{n}=0.01 \mathrm{~m}$, a constant. The phase velocity is numerically calculated inserting all the $\Delta t_{n}$ computed with $\Delta r_{n}=0.01 \mathrm{~m}$ in equation 33 . Panel (b) of Figures 11 and 12 compares the numeric and analytical results for the absolute value of the phase velocity $v_{\mathrm{f}}$ for respectively the wave amplitudes $\psi_{o+}=0.1$ and $\psi_{o+}=0.2$ described above. The analytical result for the phase velocity $v_{\mathrm{f}}$ uses the expression described in equation (27). Nice agreement can be observed. 


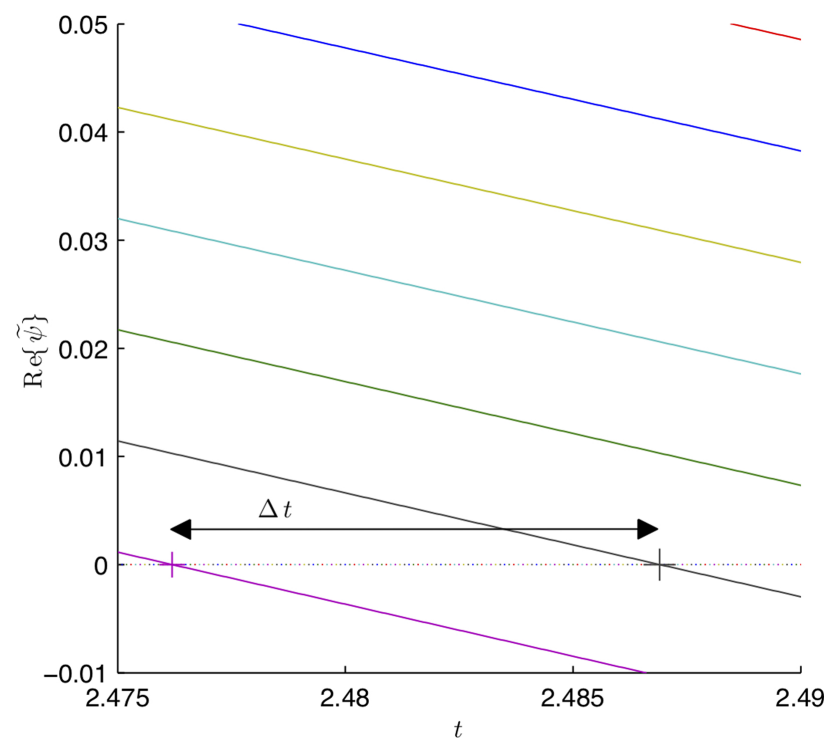

Figure 13: A detailed description of the crossing times by $\widetilde{\psi}=0$ of the first two waves plotted in Figure 11 . The difference between this consecutive crossing times is exactly the time interval $\Delta t$ used to evaluate the phase velocity in this specific interval.

\section{Conclusions}

The superposition of plane waves in a physical medium can produce a resultant wave with a phase velocity distinct from the former plane wave components. Such is the case of a wave incident obliquely over an infinite and perfect conducting surface, in which the phase velocity of the resultant wave in the incident medium becomes also - although it remais constant - a function of the incidence angle.

In the present situation of counterpropagating waves, it was demonstrated that the superposition of plane waves can also produce a resultant wave with phase velocity that depends of the spatial coordinate $r$ or the time $t$, depending of the approach adopted. As shown, both approaches are equivalent, since they provide results that are identical to each other when the same reference phase is considered.

Considering the temporal approach, mathematically, the phase velocity of the resultant wave could be expressed as $\widetilde{v}_{\mathrm{f}}=v_{\mathrm{f}}^{\text {plane }} \cdot f\left(\psi_{o+}, \psi_{o_{-}}, t\right)$, in which $v_{\mathrm{f}}^{\text {plane }}=$ $\omega / \beta$ represents the absolute value of the well-known phase velocity expression for the plane waves, such as $\psi_{+}$and $\widetilde{\psi}_{-}$are in this work. The dimensionless factor $f$ accounts the propagative effects - resultant of the superposition of counterpropagating waves - in the present case, which is a function of the amplitudes of the individual waves, $\psi_{+}$and $\psi_{-}$, and the time $t$. It must be also observed that, although $\widetilde{v}_{\mathrm{f}}$ is function of time $t,\left\langle\widetilde{v}_{\mathrm{f}}\right\rangle$ is a constant exactly equal in magnitude to $v_{\mathrm{f}}^{\text {plane }}$. This is physically reasonable, since in this case there is no changes in the physical medium or boundaries, which are generally the mechanisms responsible to effectively impact the phase velocity. Over a period $2 \pi / \omega$, the resultant wave $\psi$ propagates in the same way as the individual waves $\psi_{+}$and $\widetilde{\psi}_{-}$alone in the physical medium.

One interesting feature identified in this work is associated with the fact of the phase velocity of the resultant wave to depend of the individual wave amplitudes. This interesting feature can be explored in electromagnetics as will be right now discussed. In charged particle accelerators, resonant interaction between charged particles and the generated electromagnetic waves is intended to cause particle acceleration. If the resultant wave of the system studied in this work is electromagnetic and propagates in a plasma, the resonant interaction of the resultant wave with the charged particles of the medium can be controlled by the amplitudes of the individual waves. More, by counterpropagation, superluminal waves can lower down its phase velocities to interact resonantly with charged particles of the medium.

This mechanism identified in this work is relevant to develop new charged particle acceleration concepts and structures. The controlling feature of the phase velocity of the resultant wave through the individual amplitudes of the plane wave components is practical from the implementation point of view. Although the amplitude control can occur through two distinct generators, it is more suitable to establish counterpropagation through reflection. In this way, instead of controlling individually the amplitude of the counterpropagating waves, one can just control the reflection coefficient, and then so the phase velocity of the resultant wave for accelerating purposes.

By the end, the analytical expressions in both representations predict the well-know results of the limit situations involving the amplitudes of the individual waves. Perfect agreement of both representations with the numerical simulations is also found.

\section{Future works}

Future works will explore this amplitude-dependant characteristic of the phase velocity of the resultant wave in the wave-particle interaction phenomena in a electromagnetic system. For that, counterpropagating waves will be considered to evolve in a plasma, and the resonant interaction between the resultant wave and the charged particles of the medium will be analysed. An analytical description of how the individual counterpropagating wave amplitudes control the resonant interaction is expected to be obtained.

\section{References}

[1] J.M. Serra, M.C. Brito, J.M. Alves and A.M. Vallera, Eur. J. Phys. 25, 5 (2004).

[2] A.C.F. Santos, W.S. Santos and C.E. Aguiar, Eur. J. Phys. 34, 3 (2013).

[3] R.K. Wangsness, Am. J. Phys. 49, 10 (1981). 
[4] S. Sefati, I. Neveln, M.A. MacIver, E.S. Fortune and N.J. Cowan, in Proceedings of the 4th IEEE RAS/EMBS International Conference on Biomedical Robotics and Biomechatronics, (IEEE RAS/EMBS, Rome, 2012), p. 1620 .

[5] O.M. Curet, N.A. Patankar, G.V. Lauder and M.A. MacIver, J. R. Soc. Interface 8, 1041 (2011).

[6] A. Grinenko, C.K. Ong, C.R.P. Courtney, P.D. Wilcox and B.W. Drinkwater, Appl. Phys. Lett. 101, 233501 (2012).

[7] S. Pitois, J. Fatome and G. Millot, Opt. Express 16, 6646 (2008).

[8] L. Bino, J.M. Silver, S.L. Stebbings and P. Del'Haye, Scientific Reports 7, 43142 (2017).

[9] M. Grenier, H.R. Jauslin, C. Klein and V.B. Matveev, Journal of Mathematical Physics 52, 082704 (2011).

[10] C. Martel, E. Knobloch and M. Vega, Physica D: Nonlinear Phenomena 137, 94 (2000).

[11] C.A. Balanis, Advanced Engineering Electromagnetics (John Wiley \& Sons, New York, 1989), 1st ed.

[12] R.E. Gibbs, The Physics Teacher 36, 2 (1998).

[13] J.M. Blair, Am. J. Phys. 50, 8 (1982).

[14] D.K. Cheng, Field and Wave Electromagnetics (AddisonWesley, New York, 1989), 2nd ed.

[15] S. Ramo, J.R. Whinnery and T. van Duzer, Fields and Waves in Communication Electronics (John Wiley \& Sons, New York, 1993), 3rd ed.

[16] C.T.A. Johnk, Engineering Electromagnetic Fields and Waves (John Wiley \& Sons, New York, 1988).

[17] C.K.C. Lieou, Eur. J. Phys. 28, 4 (2007).

[18] J.D. Jackson, Classical Electrodynamics (John Wiley \& Sons, New York, 1998), 3rd ed.

[19] J.R. Reitz, F.J. Milford and R.W. Christy. Foundations of Electronmagnetic Theory (Addison-Wesley, New York, 1979), 3rd ed. 